\title{
Railway Track Recognition Based on Radar Cross-Section Statistical Characterization Using mmWave Radar
}

\author{
Shuo Li ${ }^{1, * \mathbb{C}}$, Jieqiong Ding ${ }^{1}$, Weirong Liu ${ }^{2}$, Heng $\mathrm{Li}^{2}$, Feng Zhou ${ }^{1}$ and Zhengfa Zhu ${ }^{1}$ \\ 1 School of Electrical and Information Engineering, Changsha University of Science \& Technology, \\ Changsha 410114, China; dingjieqiong@stu.csust.edu.cn (J.D.); zhoufengcsu@csust.edu.cn (F.Z.); \\ zhuzhengfa@csust.edu.cn (Z.Z.) \\ 2 School of Computer Science and Engineering, Central South University, Changsha 410083, China; \\ frat@csu.edu.cn (W.L.); liheng@csu.edu.cn (H.L.) \\ * Correspondence: lishuo@csust.edu.cn; Tel.: +86-139-0846-8594
}

check for updates

Citation: Li, S.; Ding, J.; Liu, W.; Li, H.; Zhou, F.; Zhu, Z. Railway Track Recognition Based on Radar Cross-Section Statistical Characterization Using mmWave Radar. Remote Sens. 2022, 14, 294. https://doi.org/10.3390/rs14020294 Academic Editors: Silvia Liberata Ullo, Alfonso Farina, Yu Yao, Harun Taha Hayvaci and Addabbo Pia

Received: 15 October 2021

Accepted: 4 January 2022

Published: 10 January 2022

Publisher's Note: MDPI stays neutral with regard to jurisdictional claims in published maps and institutional affiliations.

Copyright: (C) 2022 by the authors. Licensee MDPI, Basel, Switzerland. This article is an open access article distributed under the terms and conditions of the Creative Commons Attribution (CC BY) license (https:/ / creativecommons.org/licenses/by/ $4.0 /)$.

\begin{abstract}
The track settlement has a great influence on the safe operation of high-speed trains. The existing track settlement measurement approach requires sophisticated or expensive equipments, and the real-time performance is limited. To address the issue, an ultra-high resolution track settlement detection method is proposed by using millimeter wave radar based on frequency modulated continuous wave (FMCW). Firstly, by constructing the RCS statistical feature data set of multiple objects in the track settlement measurement environment, a directed acyclic graph-support vector machine (DAG-SVM) based method is designed to solve the problem of track recognition in multiobject scenes. Then, the adaptive chirp-z-transform (ACZT) algorithm is used to estimate the distance between the radar and the track surface, which realizes automatic real-time track settlement detection. An experimental platform has been constructed to verify the effectiveness of the proposed method. The experimental results show that the accuracy of track classification and identification is at least $95 \%$, and the accuracy of track settlement measurement exceeds $0.5 \mathrm{~mm}$, which completely meets the accuracy requirements of the railway system.
\end{abstract}

Keywords: millimeter wave radar; radar cross section (RCS); target recognition; frequency-modulated continuous wave (FMCW); statistical feature extraction; support vector machine (SVM)

\section{Introduction}

High-speed trains have extremely high requirements for the smoothness of the tracks. The track subsidence causes a decrease in flatness and makes the train unable to reach the designed speed. In more terrible cases, the track subsidence has been one of the main causes of serious accidents for high-speed train, such as the derailment of Eurostar train running at $300 \mathrm{~km} / \mathrm{h}$ in June 2000 [1]. However, the traditional manual track settlement detection methods, such as precision leveling and precise trigonometric leveling, are difficult for large-scale settlement detecting and automatic monitoring [2]. The detection methods based on interferometric synthetic aperture radar (InSAR) and three-dimensional laser, have the weak anti-interference ability or the expensive equipment cost [3-5].

Recently, it has been promising to use millimeter FMCW radar on the track settlement measurement. As a robust and cost-effective technology, FMCW radar can ensure the safety of high-speed rail operations by real-time measurement of track settlement. With the non-contact mode, FMCW radar does not raise the side effects on the target, and can work in harsh applications such as dust, darkness, and high temperatures circumstances. Furthermore, its sensing mechanism allows simultaneous distance measurement of multiple targets with guaranteed accuracy.

Despite its attractive application prospects, two fundamental issues should be addressed for the settlement detection of railway tracks. Firstly, the actual track settlement detection scene is a multi-objective scene. How to accurately and timely recognize the track 
among the multiple targets in the scene must be solved. Secondly, the radar can detect the settlement of the track by calculating the change in the distance between itself and the track. The distance estimation between the radar and the track needs to be accurate enough to meet the requirement of settlement measurement [6].

To address the above issues, a target recognition method is proposed based on the RCS statistical data set under low bandwidth requirement of $1.5 \mathrm{GHz}$. It is more suitable for applications in embedded sensors with limited computing resources. High accuracy can be achieved owing to the advanced super-resolution frequency estimation based on ACZT algorithm. The main contributions are summarized as follows:

- $\quad$ The RCS data set of track settlement detection under the actual scenario is constructed. The statistical feature extraction and analysis method based on RCS data set is proposed, and the multi-class DAG-SVM based recognition algorithm is applied to achieve accurate track recognition in a dynamic and static mixed multi-object scene.

- The ACZT algorithm is adopted to achieve the high-precision distance estimation between the track and radar for complex multi-target scenarios under the requirement of lower bandwidth $1.5 \mathrm{GHz}$, realizing the distance estimation accuracy of $0.5 \mathrm{~mm}$.

- A complete and feasible high-precision detection method for track settlement is designed, and the practical experiment platform is established to verify the proposed method by using the common commercial-grade millimeter-wave radar. Simultaneously, the influence of different track condition on the detection performance has been investigated thoroughly.

The remainder of this article is organized as follows: Section 2 reviews the related work. Section 3 presents the system framework of track settlement detection; Section 4 introduces the collected RCS data and its statistical features and applies the DAG-SVM based classification algorithm to achieve RCS-based track recognition; Section 5 analyzes the intermediate frequency (IF) signal to obtain a high-resolution frequency, so the high precise track settlement detection is completed; Section 6 constructs an experimental platform and conducts numerous experiments to verify the proposed method and explore the effect of various factors.

\section{Relatred Work}

For two-faced problems: i.e., (i) recognizing the track in scenarios, and (ii) measuring the distance between the radar and track precisely, we respectively review the applications of recognition approaches and distance measurement methods based on millimeter-wave radars.

Track recognition is the first step for track settlement detection. Radar cross section (RCS) statistical characteristics is important for radar-based recognition [7]. In [8,9], the current techniques used to monitor small unmanned aerial vehicles (UAVs) or drones based on radar RCS characteristics are explored. In $[10,11]$, researchers use the least-squares method to fit the RCS distribution probability function of the object and construct a multiclassification classifier to realize the recognition of people, vehicles, drones and other targets. In [12], a set-valued model of radar target recognition problem was established, and the cone frustum target recognition was realized based on this model.

Moreover, literature [13] realized the recognition of five road structures in the experimental radar system based on RCS statistical characteristics. In addition to the identification based on RCS characteristic data, literature [14,15] realized the dynamic identification of pedestrians and vehicles in the FMCW radar system through phase characteristics or amplitude characteristics binary. It is worth mentioning that the target recognition algorithm in existing studies [14-16] mainly focus on dynamic targets. It is still a challenge to recognize both the dynamic and static targets. Following their work, we set up a mixed experimental scene of dynamic and static targets. We construct RCS statistical characteristic data sets and apply machine learning methods to realize multiclass classification. 
With the track recognition, the distance measurement is another critical task for track settlement detection. FMCW radar sensors use multiple frequencies to obtain distance information of one or more targets $[17,18]$. For short distance ranging, combined with a tunable FIR filter, FMCW radar can correctly detect distance and small displacement in a multitarget environment [19]. By utilizing a $24 \mathrm{GHz}$ bandwidth, frequency and phase can be combined to estimate the ultra-high-resolution range of radar reflection angle targets [20]. S. Scherr et al. proposed a high-precision distance estimation algorithm for FMCW radar based on chirp-z-transform (CZT) [21,22]. Literature [23] proposed a continuous-wave (CW) radar based on a millimeter-wave six-port interferometer to measure the distances and Doppler frequencies with high accuracy. Bhutani et al. studied the effect of different frequencies on the ranging accuracy of the FMCW radar using the Cramér-Rao lower bound (CRLB) [24]. The current FMCW radar ranging requires high hardware requirements, and real-time performance is still limited. Their work carries out useful exploration on highprecision distance measurement of millimeter-wave radar and inspires us to develop millimeter-wave radar for railway track settlement measurement.

\section{System Framework and Problem Formulation}

The block diagram of the railway track settlement measurement system based on millimeter-wave radar is shown in Figure 1. The system's process is first to use FMCW radar to collect the raw data in the settlement measurement scene and perform data processing. Then, the processed data is used to train the classifier to complete the track recognition task. Finally, according to the corresponding relationship between frequency and distance in the raw data, track settlement detection is realized. The system consists of two modules: track recognition module and track settlement measurement module.

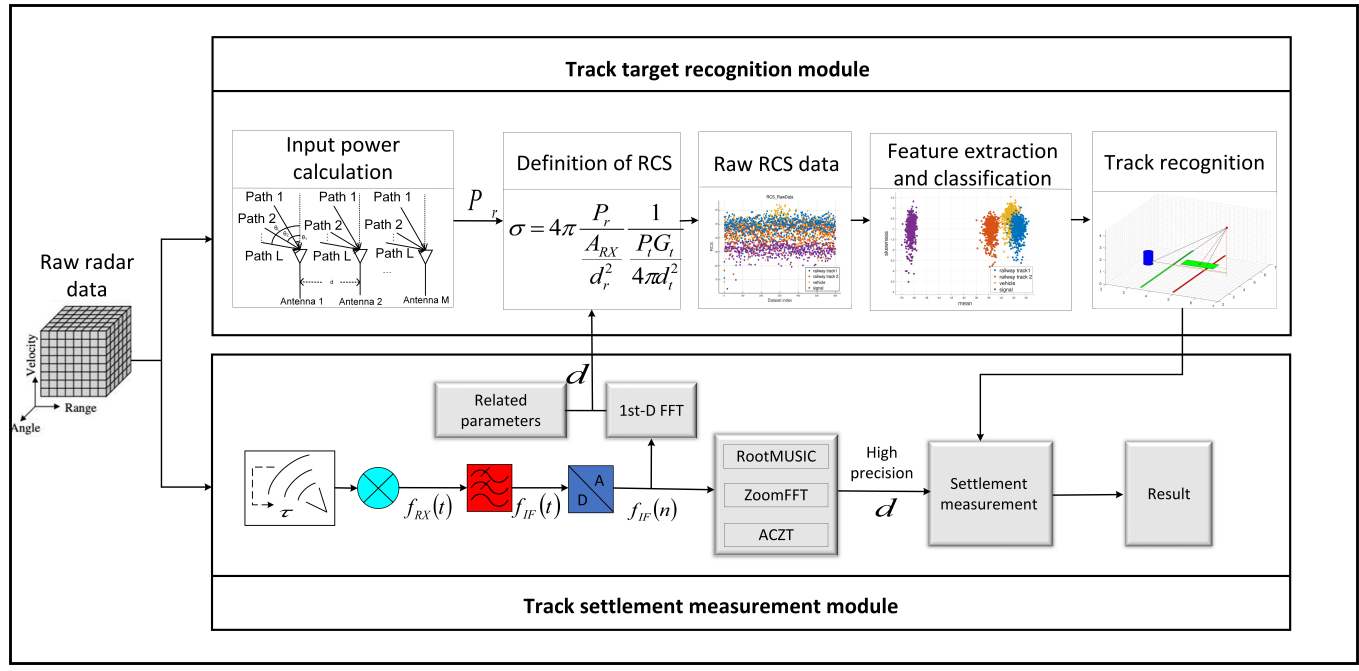

Figure 1. Railway track settlement measurement system based on millimeter wave radar.

Track recognition module. The track recognition module performs accurate and rapid recognition of track in multi-object scenes. The module calculates and collects each object's RCS value according to the reflected signal's strength, the approximate distance $d$ of the object, and the millimeter-wave radar's parameters. Furthermore, it constructs the raw RCS data set. Then, statistical methods are used to extract features from the raw RCS data set. After simulation experiments and data analysis, the four features of mean, variance, skewness, and kurtosis were finally selected as input data to train DAG-SVM and classifiers, to achieve track recognition based on target classification. 
Track settlement measurement module. Based on the target (track) determined by the track recognition module, the signal processing module detects the track settlement by monitoring the change in the distance between the radar and the track in real-time. The track settlement measurement module uses FMCW technology to convert the object's distance estimation into the frequency estimation of the IF signal. The radar's signal is transmitted to the measurement target and then reflected to form a radar echo. The radar echo is received by the radar receiving antenna. The radar echo and the transmitted wave pass through the mixer to generate a mixed signal. The mixed signal is filtered and amplified and then output to the IF signal. Finally, In order to meet the accuracy requirements of track settlement detection, ultra-high-resolution frequency estimation algorithms (ACZT) are used.

In this scenario, to realize the measurement of track settlement, the first problem that needs to be solved is to identify the track from other objects accurately, and secondly, the distance between the radar and the track needs to be accurately calculated. According to the principle of radar ranging, the key to distance estimation is a high-resolution estimation of frequency. The focus of frequency estimation is mainly on two aspects: the algorithm's requirements for the accuracy of frequency estimation and the requirement of the calculation amount of the algorithm. Real-time high-precision track settlement measurement puts high demands on both aspects, which is a difficult challenge.

\section{Track Recognition Based on RCS Statistical Feature Data Set and Classifier}

\subsection{Definition and Influencing Factors of RCS}

Radar cross section (RCS) is a physical quantity to measure the echo intensity of the target under the radar irradiation [25]. From the point of view of radar observation, the definition of RCS is given:

$$
\sigma=4 \pi \frac{P_{r}}{\frac{A_{R X}}{d_{r}^{2}}} \frac{1}{\frac{P_{t} G_{t}}{4 \pi d_{t}^{2}}}
$$

where $P_{r}$ and $P_{t}$, respectively, denote the receiver's input and the transmitter's output, $G_{r}$ and $G_{t}$, respectively, denote the gain of the receiving antenna and the gain of the transmitting antenna, $d_{t}$ and $d_{r}$, respectively, denote the distance of the receiving antenna and the transmitting antenna to the target. $A_{R X}$ is the effective aperture area of the receiving antenna and the expression is $A_{R X}=\frac{G_{r} \lambda^{2}}{4 \pi}$, where $\lambda$ represents the wavelength.

RCS $\sigma$ can be estimated according to the known parameters $\left(P_{r}, P_{t}, G_{r}, G_{t}, d_{t}, d_{r}\right.$, $\left.A_{R X}\right)$ in Equation (1) and influenced by these factors. Furthermore, the polarization of the incident electromagnetic waves, surface material, and other factors will also affect the target's RCS estimation [26,27]. In particular, when the target is radiated from the same frequency electromagnetic wave from different directions, its RCS is different. Therefore, as a physical feature, RCS is applied to recognize different targets in multi-target scenarios.

\subsection{RCS Data Set Construction and Statistical Feature Extraction}

Targets in the environment were scanned by the radar at the total chirp time of $660 \mu \mathrm{s}$ (idle time $=560 \mu \mathrm{s}$, chirp time $=100 \mu \mathrm{s}$ ), and the RCS of each target was calculated. The total scan time lasted $330 \mathrm{~ms}$, and the number of RCS samples collected per target was 600. In Figure 2, the RCS data of four targets (track 1, track 2, vehicle, signal machine) are displayed in different colors. Whether the object is stationary or in motion (vehicle), the same RCS value may correspond to different targets, and the RCS value of the same target is not a constant, but randomly fluctuates within a certain range. Therefore, target recognition cannot be completed through raw RCS data. It is necessary to reconstruct the RCS data and extract their features to recognize the track based on RCS in the multi-object scenario. 


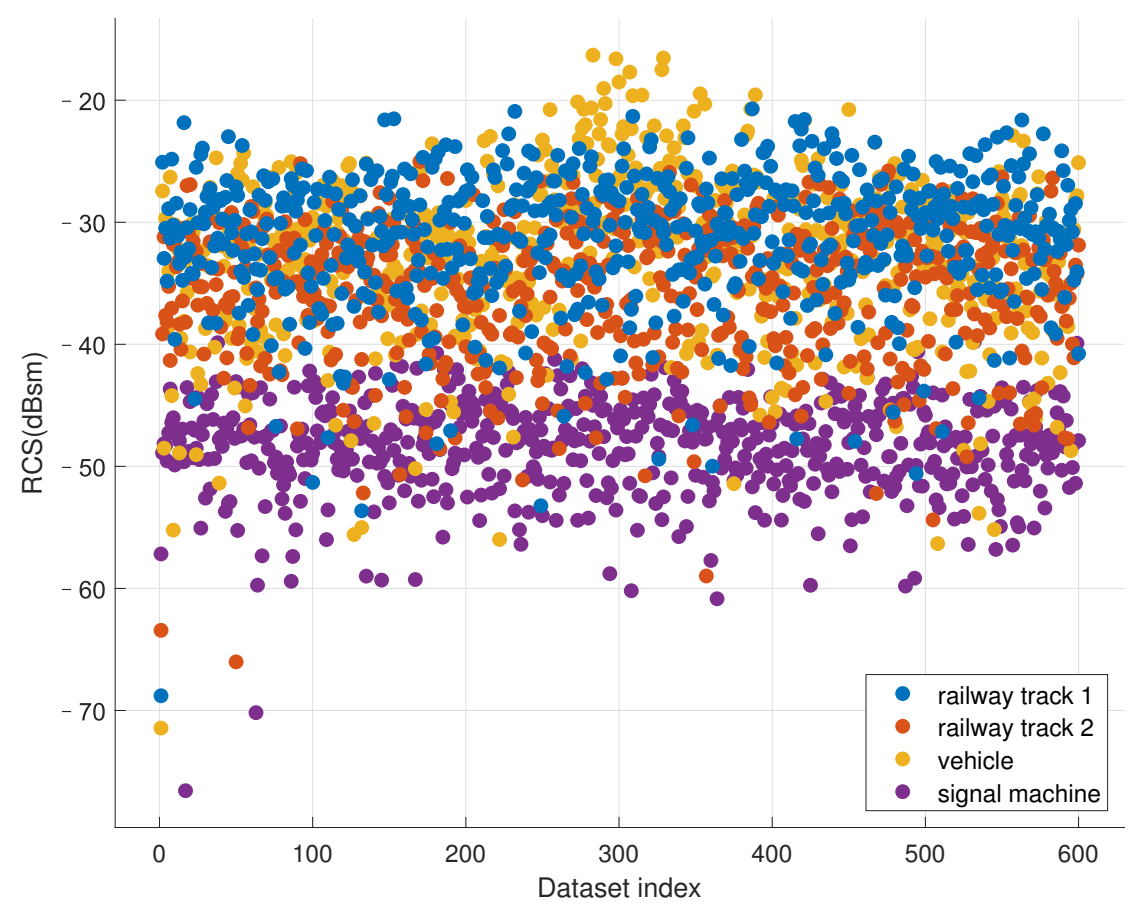

Figure 2. A large number of RCS measurement raw data collected in the experimental environment.

Set the chirp time of the millimeter wave radar to $1 \mu \mathrm{s}$, and a frame contains 600 chirps, $N=600$. For each target, a dataset containing 600 temporal RCS samples is obtained in per frame. For example, when the millimeter wave radar transmits a frame, and the set of temporal RCS samples for target $k$ is denoted by $\mathbf{p}_{k}^{(n)}=\left[p_{k}^{(1)}, \ldots \ldots, p_{k}^{(N)}\right]$. Through discrete wavelet transform of raw RCS data $\mathbf{p}_{k}^{(n)}$, we can obtained:

$$
\begin{aligned}
& \mathbf{A}(i, j)=\sum_{n=1}^{N} \mathbf{p}_{k}^{(n)} \psi\left(\frac{n-j}{i}\right) \\
& i=1,2, \ldots, N ; j=1,2, \ldots, L
\end{aligned}
$$

where $\psi\left(\frac{n-j}{i}\right)$ is the wavelet basic function determined by $i$ and $j, L$ is the number of features. Mathematically, $\mathbf{A}(i, j)$ can be expressed as $\mathbf{A}(i, j)=\left[\mathbf{f}_{n}(1), \mathbf{f}_{n}(2), \ldots, \mathbf{f}_{n}(N)\right]$, where $\mathbf{f}_{n}=\left[f_{k, n}^{(1)}, \ldots \ldots, f_{k, n}^{(L)}\right]$. Obviously, $\mathbf{A}(i, j)$ is still too enormous to be used directly. Thus, through extensive observation of RCS datasets of different targets, four features (the mean $\mu_{n}$, the variance $h_{n}$, the skewness $g_{n}$, and the kurtosis $u_{n}$ ) of the RCS dataset are applied to recognize different targets. The expression of the four features are given by:

$$
\begin{aligned}
\mu_{n} & =\frac{1}{L N} \sum_{i=1}^{L} \sum_{j=1}^{N}|\mathbf{A}(i, j)| \\
h_{n} & =\frac{1}{L N} \sum_{i=1}^{L} \sum_{j=1}^{N}\left[|\mathbf{A}(i, j)|-\mu_{n}\right]^{2} \\
g_{n} & =\frac{\frac{1}{L N} \sum_{i=1}^{L} \sum_{j=1}^{N}\left[|\mathbf{A}(i, j)|-\mu_{n}\right]^{3}}{\left\{\sqrt{\frac{1}{L N} \sum_{i=1}^{L} \sum_{j=1}^{N}\left[|\mathbf{A}(i, j)|-\mu_{n}\right]^{2}}\right\}^{3}} \\
u_{n} & =\frac{\frac{1}{L N} \sum_{i=1}^{L} \sum_{j=1}^{N}\left[|\mathbf{A}(i, j)|-\mu_{n}\right]^{4}}{\left\{\frac{1}{L N} \sum_{i=1}^{L} \sum_{j=1}^{N}\left[|\mathbf{A}(i, j)|-\mu_{n}\right]^{2}\right\}^{2}}
\end{aligned}
$$


The calculation process of the statistical features can be realized by arithmetic operation, and it takes about $40 \mathrm{~ms}$. It can complete 25 calculations per second and transmit to the observation platform, which is operable for the experimental radar system to monitor the target in real-time. Therefore, the statistical feature datasets $\mathbf{f}_{n}$ is composed of the mean $\mu_{n}$, variance $h_{n}$, skewness $g_{n}$ and kurtosis $u_{n}$ in Equation (3), and a scatter plot of four statistical features is shown in Figure 3. The area covered by different statistical features is roughly distinguishable. However, which type of target the points in the overlapping area belong to is ambiguous. Therefore, more effective recognition algorithms are needed to achieve accurate target recognition. We use the DAG-SVM and decision tree methods to design the classifier for track recognition.

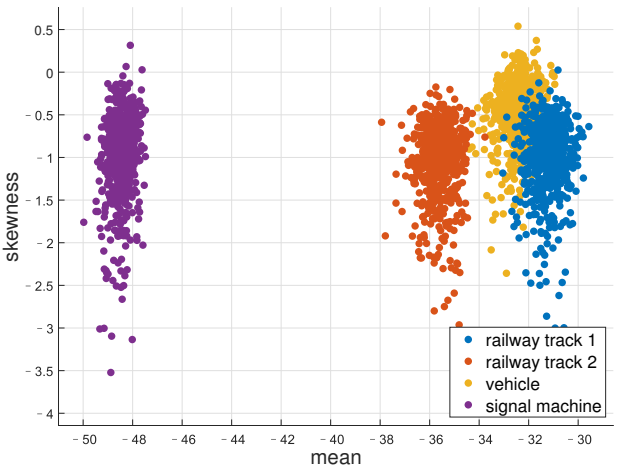

(a)

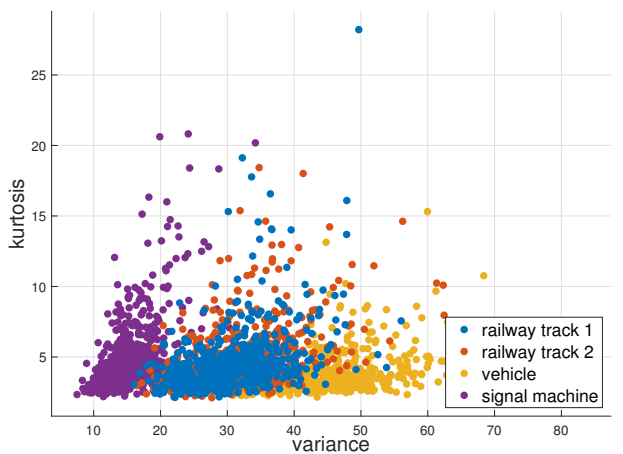

(c)

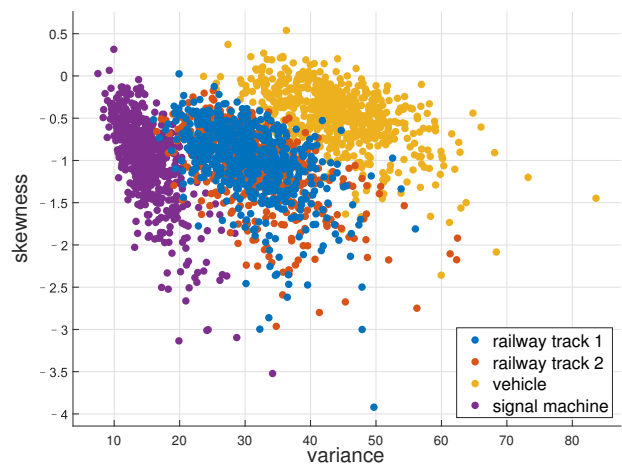

(b)

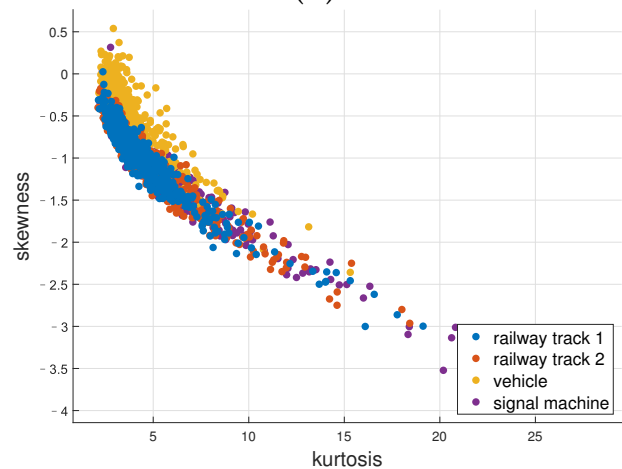

(d)

Figure 3. Overlapping areas between two statistical features. (a) $x$ - mean $y$-skewness, (b) $x$-variance $\mathrm{y}$ - skewness, (c) $\mathrm{x}$ - variance $\mathrm{y}$ - kurtosis, (d) $\mathrm{x}$ - kurtosis $\mathrm{y}$ - skewness.

\subsection{Track Recognition Based on DAG-SVM and Decision Tree}

Based on the RCS feature datasets constructed with the four characteristics mean $\mu_{n}$, variance $h_{n}$, skewness $g_{n}$ and kurtosis $u_{n}$ in Section 4.2, DAG-SVM is applied to complete the classification task [28]. The algorithm's key idea is to convert the "multiclassification" into multiple "two-classification" by constructing and combining multiple two-class classifiers [29].

Suppose the input data has $C$ classes, there are $\frac{C(C-1)}{2}$ nodes and $C$ leaf nodes, where each node represents a two-class classifier and each leaf node represents a class. In this paper, $C=4$, which means the four classification categories of railway track 1 , railway track 2, vehicle, and signal machine. As shown in Figure 4, by building a threelayer classifier model (each layer contains several two-class classifiers), the test data can be divided into one of four classes. It can be seen that the classification function expression of the two-class classifier is:

$$
h\left(\mathbf{f}_{n} ; s, \mathbf{a}, b\right)=\frac{1}{s} \times \mathbf{f}_{n} \cdot \mathbf{a}^{T}+b
$$




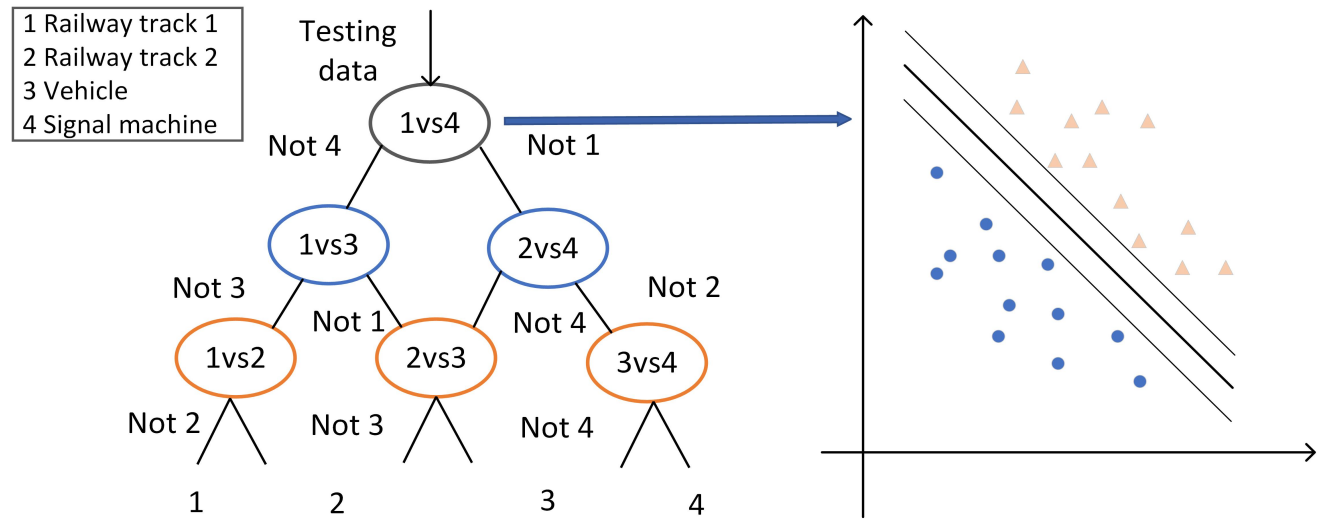

Figure 4. The decision-making process of the four-class classifier based on the DAG-SVM method.

According to Section $4.2, \mathbf{f}_{n}=\left[\mu_{n}, h_{n}, g_{n}, u_{n}\right]$, which is the feature set corresponding to the four statistical features (mean $\mu_{n}$, variance $h_{n}$, skewness $g_{n}$ and kurtosis $u_{n}$ ) obtained in the $n$-th scan of the radar. $s$ denotes the scale parameter, a denotes the hyperparameter vector, and $b$ denotes the bias parameter, which are applied to adjust the appropriate classification function. We train the DAG-SVM model with the feature data set collected in the experiment to obtain the corresponding values of $s, \mathbf{a}$, and $b$. Therefore, a classification function is predetermined based on the feature data set and serves as a decision boundary. Based on this functionality, a given feature set can be typically divided into two distinct regions. In this way, the multiple two-class classifiers are designed.

We use a five-fold cross-validation method to train and test the DAG-SVM model. The feature data set is divided into five subsets, one of which is used to test the model, and the other four subsets are known as the training set. In this method, the parameter with the smallest estimation error of the test set is considered as the best one.

\section{Track Settlement Measurement Based on FMCW Technology with High Precision Target Distance Estimation}

On the basis of the railway track recognition, ACZT algorithm provides high-resolution frequency to accurately estimate the distance between the radar and the railway track, to realize railway track settlement measurement.

\subsection{Basics of FMCW Radar Operation and Range Estimation}

The Basics of FMCW radar operation and range estimation is shown in Figure 5. The transmitted signal is given by:

$$
f_{T X}(t)=e^{j\left(2 \pi f_{s} t+\frac{\pi B}{T_{\text {sweep }}} t^{2}\right)}
$$

where $B$ is the bandwidth and $T_{\text {sweep }}$ is the chirp time. The received signal after the propagation delay $\tau$ is represented by:

$$
f_{R X}(t)=e^{j\left(2 \pi f_{s}(t+\tau)+\frac{\pi B}{T_{\text {sweep }}}(t+\tau)^{2}\right)}
$$

After the transmitted signal and received signal pass through the mixer, the immediate frequency(IF) signal from an object at range $d$ is given by:

$$
f_{I F}(t)=e^{j\left(4 \pi \frac{B d}{c T_{\text {sweep }}} t+\frac{4 \pi}{\lambda} d\right)}
$$




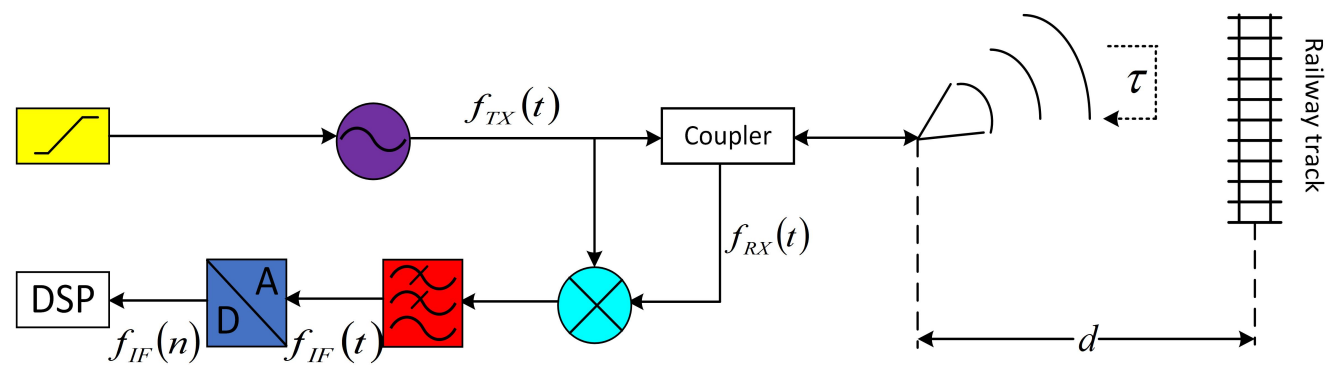

Figure 5. Block diagram of the FMCW radar system.

The target distance calculation is converted to estimate the corresponding IF signal frequency. Since the radar measures multiple points of targets simultaneously, the IF signal contains the distance information of multiple points. Therefore, the 1D-FFT is used to obtain the range profile, and the actual spectrum peak position is determined according to the range profile. That is to say; the spectrum peak point serves as the detection point of the radar target. In the next step, the ACZT algorithm performs frequency estimation of the peak point.

\subsection{High Precision Distance Estimation Based on ACZT Algorithm}

CZT is an extension of FFT transform calculated in z plane. $x(n)$ is the sampling sequence of the IF signal $f_{I F}(t)$; the peak of the FFT spectrum provides the $f_{I F_{-} F F T}$ value. Besides, the frequency resolution is $\frac{f_{s}}{N}$, where $f_{s}$ and $N$ represent the sampling frequency and the number of samples, respectively. The target distance estimation resolution using the FFT algorithm is $\frac{c}{2 B}$. Obviously it is difficult to meet the high-resolution frequency estimation requirements. While CZT can sample the signal locally and perform spectrum analysis within a limited frequency range to improve frequency resolution and settlement measurement accuracy [30]. It is defined with the starting point $A$, step $W$ and resolution $M$ which is defined with number of points in frequency spectrum of $x(n)$. The mathematical expression of the CZT is given by:

$$
\begin{aligned}
& X(z)=\sum_{n=0}^{N-1} x(n) A^{-n} W^{-n K}, 0 \leq K \leq M-1 \\
& A=A_{0} e^{j \theta_{0}} ; W=W_{0} e^{j \varphi_{0}} .
\end{aligned}
$$

As shown in Figure 6, $A_{0}$ is its radius, $\theta_{0}$ represents the starting angle, $\varphi_{0}$ represents angular frequency difference between two neighboring points; $W_{0}$ controls the circumference of the circle bending inwards or outwards. The expression of the upper and lower boundary frequencies of the frequency band $f_{w}=\left[f_{L}, f_{U}\right]$ is:

$$
\begin{aligned}
& f_{L}=\frac{f_{s} \theta_{0}}{2 \pi}=f_{I F_{-} F F T}-\frac{f_{s}}{N} \\
& f_{U}=\frac{f_{s}\left(\theta_{0}+(M-1) \varphi_{0}\right)}{2 \pi}=f_{I F_{-} F F T}+\frac{f_{s}}{N}
\end{aligned}
$$

Based on the frequency sweep upper and lower bounds determined by FFT and the refinement points $M$, the frequency estimation resolution of the CZT algorithm is $\frac{f_{s}}{2 M N}$, so its range resolution is $\frac{c}{4 B M}$. Consequently, as the number of refinement points $M$ increases, the frequency resolution of CZT increases. However, the increase of $M$ brings higher computational effort, and $M$ cannot increase indefinitely within a certain frequency band $f_{w}$. Therefore, the selection of the refined frequency band $f_{w}$ determined by the parameters $A$ and $W$ is cautious. Unfortunately, the initial CZT algorithm parameters $A$ and $M$ are inconvenient to change after being determined. 


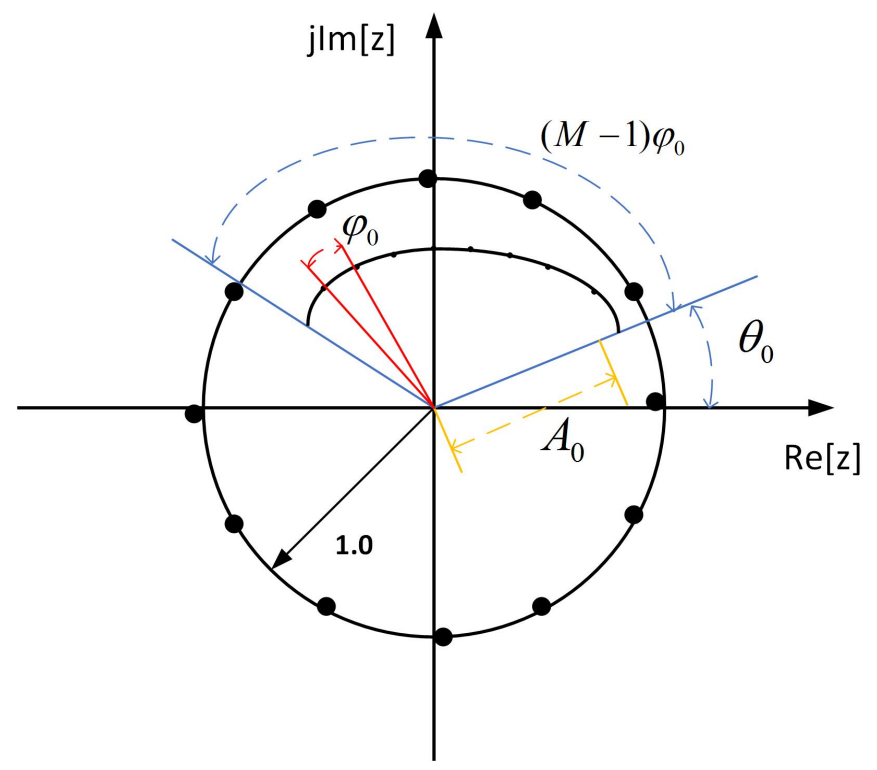

Figure 6. Z-plane unit circle representation of the CZT.

To this end, this paper adopts an adaptive CZT method that can automatically configure parameters. The algorithm aims to improve the efficiency and frequency measurement accuracy by narrowing the frequency band observed by the CZT method $\left[f_{L}, f_{U}\right]$. The ACZT algorithm flow is shown in Figure 7. Firstly, the IF signal is preprocessed by the FFT algorithm to obtain the rough frequency $f_{I F_{-} F F T}$ and the original frequency band $\left[f_{L}, f_{U}\right]$. Combining (8) and (9), we can calculate $A=e^{j 2 \pi f_{L} / f_{s}}$ and $W=e^{j 2 \pi\left(f_{U}-f_{L}\right) / M f_{s}}$. It can be seen that the calculation of parameters $A, W, M$ is closely related to $f_{L}$ and $f_{U}$. Therefore, we use an adaptive regulator to obtain better model parameters $A, W, f$, which narrows the CZT processing frequency range to $\left[f_{\min }, f_{\max }\right]$.

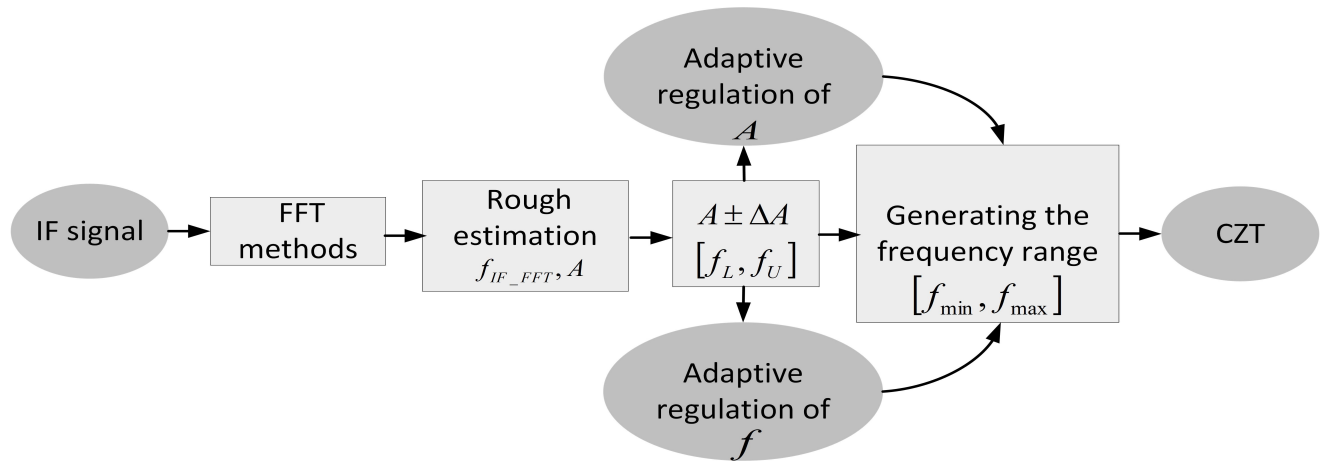

Figure 7. Algorithm flow for ACZT.

After multiple convolution operations, composite operations and exponential operations, the computational complexity of CZT on the number of points $M$ sampled in the frequency band $\left[f_{L}, f_{U}\right]$ is given by:

$$
O(L, M)=O\left(\frac{3 L}{2} \log _{2}(L)+2 L+N\right)
$$

where $L=N+M-1$, which is the total number of points that need to be calculated, and $N$ is the length of the signal sequence. Since $N \leq L$, the Equation (10) can be simplified as:

$$
O(L)=O\left(\frac{3 L}{2} \log _{2}(L)+3 L\right)
$$


After narrowing the frequency range $\left[f_{L}, f_{U}\right]$ to $\left[f_{\min }, f_{\max }\right]$ through the adaptive regulator, the number of points to be processed by CZT is reduced from $M$ to $M_{a}$. As a result, the total number of points is reduced to $L_{a}=N+M_{a}-1=\frac{L}{k}, k \geq 1$, where $k$ is the reduction factor. The algorithm complexity can be written as:

$$
\begin{aligned}
O\left(L_{a}\right) & =O\left(\frac{L}{k}\right)=O\left(\frac{3 L_{a}}{2} \log _{2}\left(L_{a}\right)+3 L_{a}\right) \\
& =O\left(\frac{3 L}{2 k} \log _{2}\left(\frac{L}{k}\right)+\frac{3 L}{k}\right) \\
& \leq O\left(\frac{3 L}{2} \log _{2}(L)+3 L\right)
\end{aligned}
$$

Since $M_{a} \leq N \leq L_{a}$, for the following algorithm complexity comparison, assuming $N=M_{a}=\frac{L_{a}}{2}$, and the reduction factor $k$ is 2 , the Equation (12) can be simplified as:

$$
O\left(L_{a}\right)=O\left(\frac{L}{k}\right)=O(2 N)=O\left(3 N \log _{2}(2 N)+6 N\right)
$$

Compared with the ACZT algorithm, the RootMUSIC algorithm extracts frequency based on the orthogonal characteristics of the noise subspace and the signal subspace. The computational complexity of RootMUSIC derives mainly from eigendecomposition. For N-point sequence $x(n)$, the computational complexity is $O\left(N^{3}\right)$. ZoomFFT retains the same resolution with a full-size FFT on the original signal by computing a small FFT on a shorter signal. The computational complexity is $O\left(\frac{N}{2} \log _{2}(N)+2 N+D N Q\right)$, where $D$ and $Q$ denote decimation factor and the digital filter order, respectively. The different computational complexity of these three are listed in Table 1.

Table 1. The computational complexity of the algorithms.

\begin{tabular}{cccc}
\hline Algorithms & ACZT & RootMUSIC & ZoomFFT \\
\hline Computation amounts & $O\left(3 N \log _{2}(2 N)+6 N\right)$ & $O\left(N^{3}\right)$ & $O\left(\frac{N}{2} \log _{2}(N)+2 N+D N Q\right)$ \\
\hline
\end{tabular}

In order to meet the requirements of measurement accuracy, the signal sequence should have sufficient length (for example, in our experiment, $N=512$ ). In this case, the complexity of the proposed algorithm is obviously lower than that of the RootMUSIC algorithm. For the ZoomFFT algorithm, to achieve the same measurement resolution as the proposed algorithm, its decimation factor $D=2 M$. According to Table 1 , the complexity of ZoomFFT is also higher than that of the proposed algorithm.

\section{Experiment}

Figure 8a shows the experimental environment of railway track settlement measurement based on millimeter-wave radar. In this environment, the experiment mainly focuses on these four objects: two tracks, a signal machine (a trackside signal device), and a track vehicle with a speed of $3.3 \mathrm{~m} / \mathrm{s}$. Among them, track 1 is the settlement detection target of the system. The millimeter-wave radar is installed vertically above track 1 . As explained in the previous chapter, the radar first uses a trained classifier to identify track 1 . On this basis, the settlement measurement is achieved by estimating the distance between the radar and track 1 with high accuracy. 


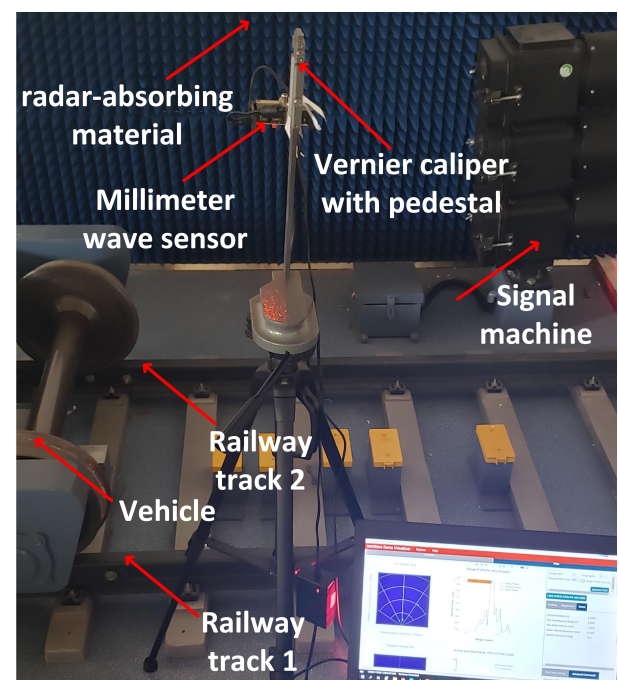

(a)

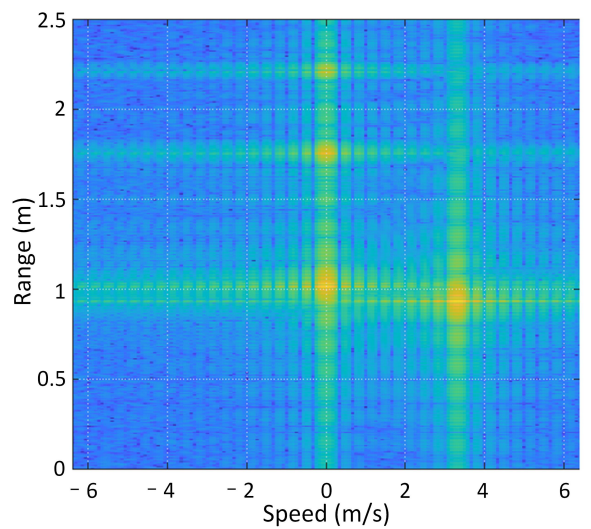

(b)

Figure 8. Photograph and range doppler heat map of the experimental environment for track settlement measurement. (a) photograph of the experimental environment; (b) range doppler heat map.

Corresponding to the radar's track settlement measurement process, we divide the experiment into two phases. In the first phase of the experiment, the track recognition algorithm's performance was evaluated, and the DAG-SVM and the decision tree algorithm with a maximum split number of 4 [31] were compared. In the second phase of the experiment, the distance estimation accuracy of ACZT, RootMUSIC [32], and ZoomFFT [33] were evaluated and compared under different distance, SNR, and vibration conditions. The parameters of the experiment are shown in Table 2.

Table 2. Experiment setting parameters.

\begin{tabular}{cc}
\hline Parameters & Value \\
\hline Starting frequency (GHz) & 77 \\
Bandwidth (GHz) & 1.5 \\
TX Antenna Gain (dB) & 36 \\
RX Antenna Gain (dB) & 42 \\
SNR (dB) & 15 \\
Number of TX Antennas used & 1 \\
Vehicle speed(m/s) & 1 \\
Number of mmwave radar ADC bits & 3.3 \\
ADC sampling frequency & $16-\mathrm{bits}$ \\
Chirp number in a frame & $5 \mathrm{MHz}$ \\
Total Chirp time(chirp time+idle time) (us) & 512 \\
& 500 \\
\end{tabular}

In the laboratory, we simulated the track settlement by changing the height of the radar. As shown in Figure 8a, the radar is fixed on the vernier caliper's measuring jaw. At the beginning of the experiment, the radar's initial height value is recorded, and then the height of the radar is adjusted by sliding the measuring jaw. At the same time, the adjusted height value is read with the vernier caliper. According to the initial height value and adjusted height value, the track settlement's actual value can be calculated. By comparing the radar's estimated settlement value with the actual settlement value, the settlement measurement accuracy of the proposed method is evaluated. 


\subsection{Performance Evaluation of Railway Track Recognition Method}

We constructed the RCS data set of each object and performed feature extraction. Two track recognition classifiers are designed using the DAG-SVM and CART decision tree methods described in Section 4.3. The CART decision tree is to find an optimal feature in the original data set through a number of judgment conditions to dichotomize and refine the data set gradually, and then recurse the above operations until the conditions for automatic classification of objects are met. Based on the RCS dataset, the experimental results of the recognition performance of different classification algorithms, distance and SNR are shown in Figures 9-11.

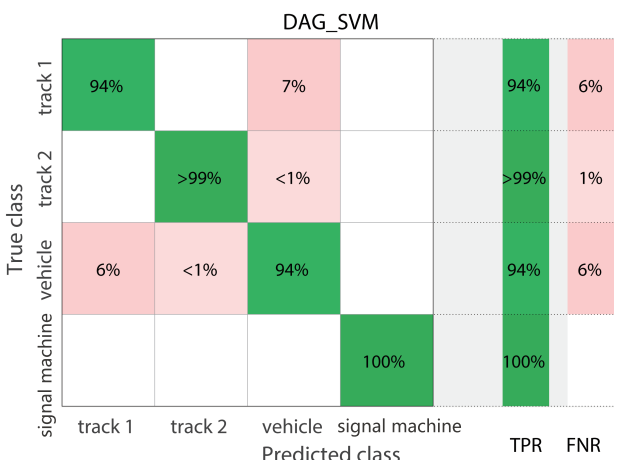

(a)

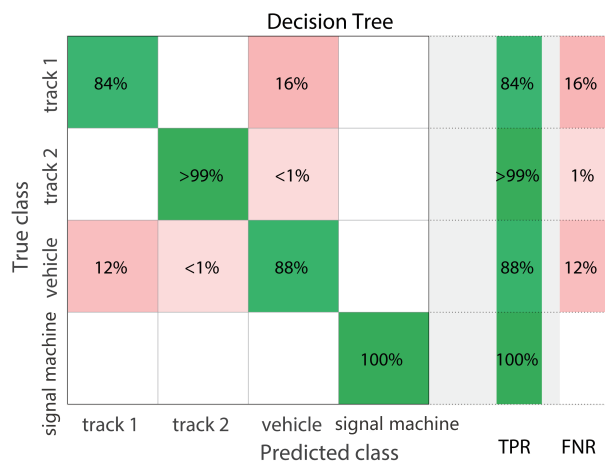

(b)

Figure 9. Confusion matrix of track recognition by DAG-SVM and decision tree at a radar height of $1.0157 \mathrm{~m}(\mathrm{SNR}=15 \mathrm{~dB})$. (a) Confusion matrix of DAG-SVM; (b) Confusion matrix of decision tree.

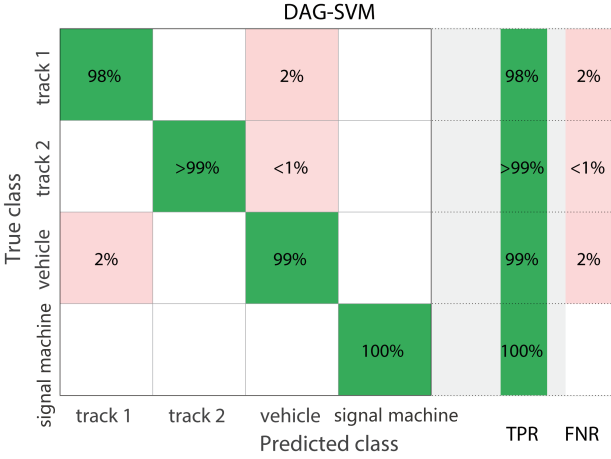

(a)

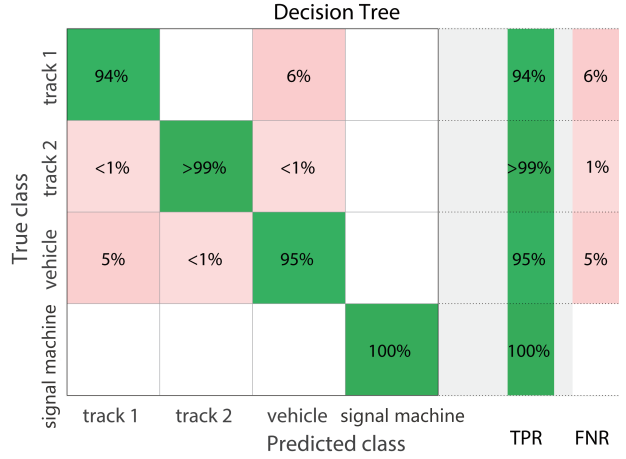

(b)

Figure 10. Confusion matrix of track recognition by DAG-SVM and decision tree at a radar height of $2.1235 \mathrm{~m}(\mathrm{SNR}=15 \mathrm{~dB})$. (a) Confusion matrix of DAG-SVM; (b) Confusion matrix of decision tree.

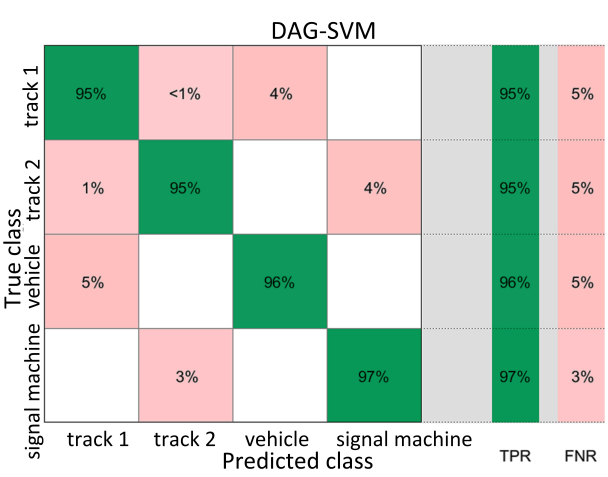

(a)

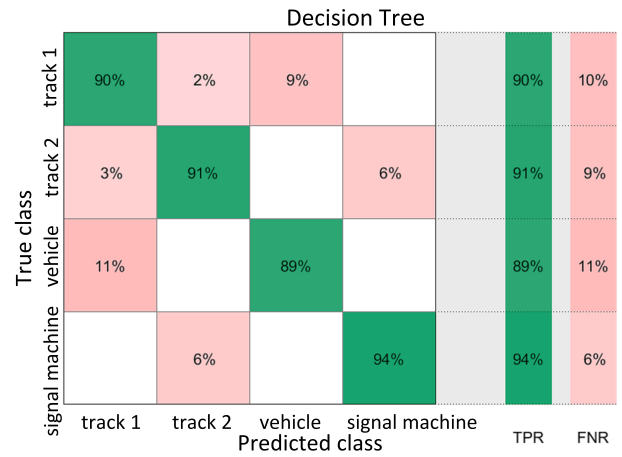

(b)

Figure 11. Confusion matrix of track recognition by DAG-SVM and decision tree at a radar height of $2.1235 \mathrm{~m}$. (SNR = 7.5 dB). (a) Confusion matrix of DAG-SVM; (b) Confusion matrix of decision tree. 
Figure 9 shows that the classification accuracy of DAG-SVM and decision tree is $96.7 \%$ and $92.9 \%$ when the height of the radar from the track 1 is $1.0157 \mathrm{~m}$. Figure 10 shows that the classification accuracy of DAG-SVM and decision tree is $98.8 \%$ and $97.0 \%$ when the height of the radar from the track 1 is $2.1235 \mathrm{~m}$. Figure 11 shows that when the SNR drops to $7.5 \mathrm{~dB}$, the classification accuracy of DAG-SVM and decision tree are $95.8 \%$ and $90.8 \%$, respectively. Obviously, the recognition accuracy of DAG-SVM is higher than that of decision tree under these three conditions. Comparing the Figures 9 and 10 we can see that the height of the radar from the track 1 will affect the accuracy of classification. This is because the change in the height of the radar will make the incident angle of the signal variable, which will affect the RCS of the target. This is the reason why track 1 and track 2 can be distinguished. In the case of a lower SNR, the recognition accuracy will also decrease.

Under the same conditions, we implement a decision tree based on the CART algorithm, which is a binary tree of cyclic analysis of the training data set composed of input variables and output variables. The input variables are composed of statistical feature data sets, and the output variables are composed of 4 different targets. The proposed CART decision tree with the maximum number of splits of 4 and the split criterion of Gini's diversity index for track recognition, and compare the performance with the classifier designed by the DAG-SVM method. The experiments also proved that DAG-SVM has higher classification accuracy than decision tree. However, as shown in Table 3, the decision tree performs better in the training time and prediction speed of the model. On the current mainstream radar embedded hardware platform, the cost of DAG-SVM is acceptable.

Table 3. Comparison of SVM and decision Tree.

\begin{tabular}{ccc}
\hline & Trainning Time & Prediction Speed \\
\hline DAG-SVM & $2.6799 \mathrm{~s}$ & $57,000 \mathrm{obs} / \mathrm{s}$ \\
Decision Tree & $0.84401 \mathrm{~s}$ & $270,000 \mathrm{obs} / \mathrm{s}$ \\
\hline
\end{tabular}

\subsection{Performance Evaluation of Railway Track Settlement Measurement Method}

As explained in the previous section, the proposed method realizes railway track settlement monitoring by accurately calculating the distance between the radar and the track. In this section, we use experiments to evaluate the accuracy of the distance estimation of the proposed method and the impact of target distance and SNR on distance estimation accuracy.

\subsubsection{Impact of the Distance between Radar and Track on Settlement Measurement}

In the experiment, we adjust the height of the radar by longitudinally sliding the measuring jaws of the vernier caliper (the height change step is $0.5 \mathrm{~mm}$ ) to change the distance between the radar and the track 1 to simulate track settlement. To discuss the accuracy of track settlement when the distance between the radar and track 1 is changeable, we conduct settlement measurements experiments when the initial distance is $1.0157 \mathrm{~m}$ and $2.1235 \mathrm{~m}$, respectively. During the experiment, the SNR is $15 \mathrm{~dB}$, and the number of test samples per distance is 500 .

Figure 12 shows the experiment results by three different algorithms when the distance between the radar and the center of track 1 is different. When the initial distance between the radar and track 1 is $1.0157 \mathrm{~m}$, as shown in Figure 12a, the measurement stability of distance estimation using the ACZT algorithm, RootMUSIC algorithm, and ZoomFFT algorithm is less than $32.8 \mu \mathrm{m}, 3.3 \mathrm{~mm}$, and $8.6 \mathrm{~mm}$, respectively. When the initial distance between the radar and track 1 is $2.1235 \mathrm{~m}$, as shown in Figure 12b, the measurement stability of distance estimation using the ACZT algorithm, RootMUSIC algorithm, and ZoomFFT algorithm is less than $65.2 \mu \mathrm{m}, 6.1 \mathrm{~mm}$, and $19.3 \mathrm{~mm}$, respectively. The experimental results show that ACZT has a better distance deviation than RootMUSIC and ZoomFFT. The experimental results also show that the shorter the distance between the radar and the 
track, the better the stability of track settlement measurement. This is because the fading of the radar signal increases as the distance increases, and the accuracy of the settlement measurement decreases.

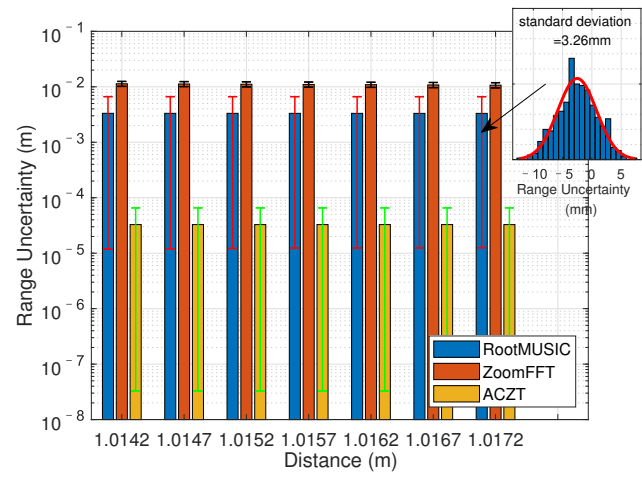

(a)

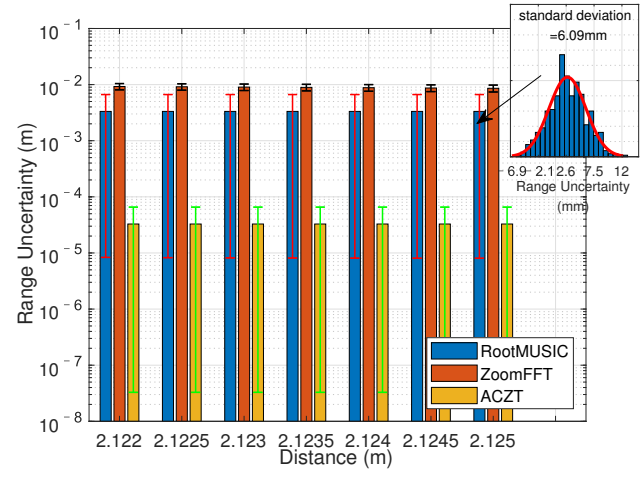

(b)

Figure 12. The experiment results by three different algorithms when the distance between the radar and the center of the track 1 is different. The height of (a) is $1.0157 \mathrm{~m}$; The height of $(\mathbf{b})$ is $2.1235 \mathrm{~m}$.

\subsubsection{Impact of the SNR on Settlement Measurement}

To evaluate the impact of noise on the accuracy of distance estimation of the proposed algorithm, in Section 6.2.2, we have designed an experiment of the measurement error vs SNR. We deployed millimeter-wave radar in the experimental scene and generated several range profiles. The SNR was computed based on the range profile as the ratio between the target's peak response and the noise floor value. The SNR collected and calculated in the experimental scenario is $35.5 \mathrm{~dB}$. Then, based on this, a simulation program is designed to add different relative power Gauss white noise to the collected signal. The measurement errors of track settlement with SNR of $35.5 \mathrm{~dB}, 28.1 \mathrm{~dB}, 26.2 \mathrm{~dB}, 23.1 \mathrm{~dB}, 19.8 \mathrm{~dB}, 17.2 \mathrm{~dB}$, $14.8 \mathrm{~dB}, 11.0 \mathrm{~dB}, 7.9 \mathrm{~dB}, 5.0 \mathrm{~dB}, 2.0 \mathrm{~dB}, 0 \mathrm{~dB},-5.1 \mathrm{~dB},-6.9 \mathrm{~dB}$, and $-19.8 \mathrm{~dB}$, were obtained, respectively. The experimental results are shown in Figure 13. In addition, the distance between the radar and track 1 is $2.1235 \mathrm{~m}$ and 500 samples are collected for each SNR point.

The distance error is calculated based on ground truth and distance estimated value, and the mean distance error and standard deviation curve are depicted in Figure 13. The settlement measurement accuracy of the ACZT, RootMUSIC, and ZoomFFT is generally improved with the increase of SNR. SNR has a significant impact on the RootMUSIC algorithm, but it follows the CRLB trend within the considered SNR range. Under the low SNR, such as $-6.9 \mathrm{~dB}$, the distance estimation error of RootMUSIC is substantial, and the average distance error is $638.4 \mathrm{~mm}$, standard deviation is $523.8 \mathrm{~mm}$. As the SNR increases, the distance estimation accuracy of the RootMUSIC algorithm continues to improve. In particular, when the SNR is higher than $35.5 \mathrm{~dB}$, the average error is $87.6 \mu \mathrm{m}$, which can meet the accuracy requirements of track settlement measurement. In contrast, SNR has a slight impact on the performance of ZoomFFT. When the SNR is higher than $2.0 \mathrm{~dB}$, the average distance error is stable at about $9 \mathrm{~mm}$ and the standard deviation is $4.2 \mathrm{~mm}$. However, at the same SNR, the average error and standard deviation of the ACZT algorithm are about $0.4 \mathrm{~mm}$. Furthermore, when the SNR is higher than $11.0 \mathrm{~dB}$, the average error is lower than $120.3 \mu \mathrm{m}$, and the standard deviation is lower than $108.7 \mu \mathrm{m}$, which is sufficient to track settlement measurement requirements. As the ACZT algorithm inherits the ability of CZT to be highly immune to interferences, the ACZT algorithm has better performance and applicability to noisy environments in this regard. 


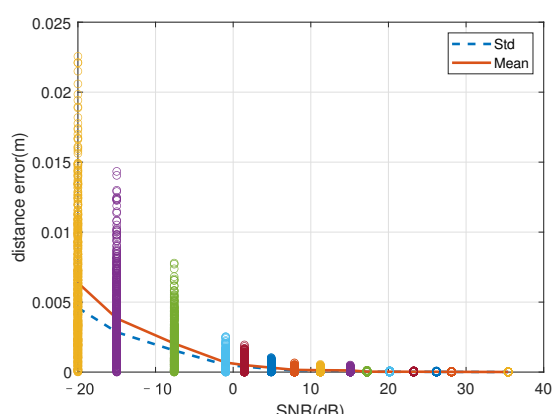

(a)

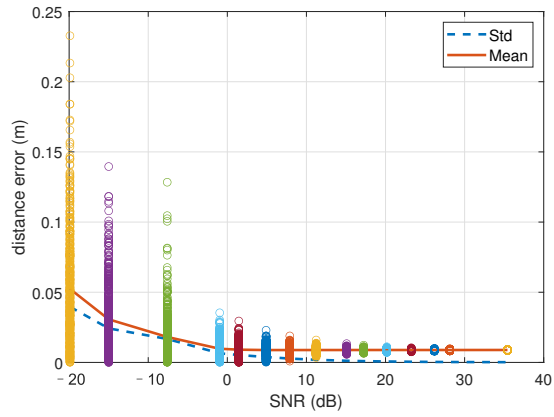

(c)

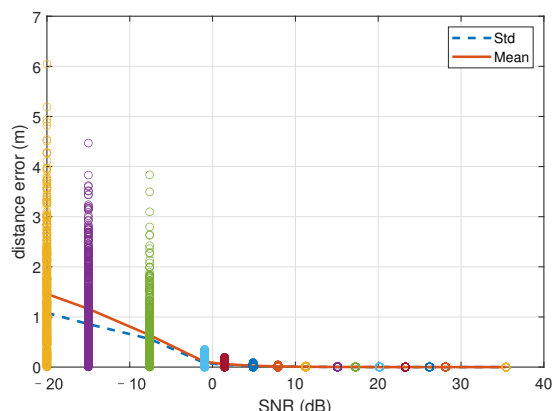

(b)

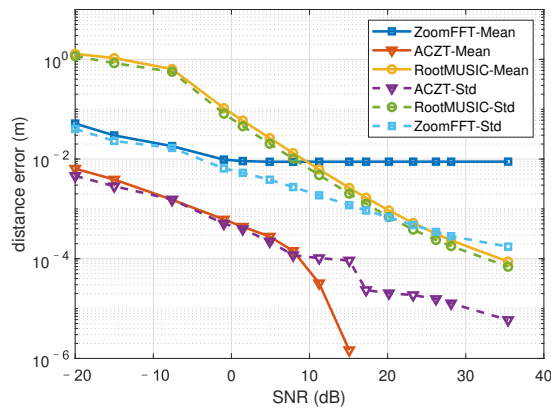

(d)

Figure 13. The distance error of three different algorithms. (a) is distance error of the ACZT; (b) is distance error of the RootMUSIC; (c) is distance error of the ZoomFFT; (d) is the comparison of distance error for three algorithms.

\subsubsection{Impact of the Vibration on Settlement Measurement}

When the high-speed train is moving, the track settlement measurement is not carried out. Nevertheless, we simulated the impact of vibration on the settlement measurement under laboratory conditions. Assuming that the train speed is $54 \mathrm{~m} / \mathrm{s}$ and the train length is $200 \mathrm{~m}$, the train passes the measuring point for $3.7 \mathrm{~s}$; that is, the vibration duration is $3.7 \mathrm{~s}$. The dominant noise at this time is wheel-rail rolling noise [34]. The track settlement data accompanied by noise is shown as the original data in Figure 14. Obviously, its settlement measurement has intense noise, and it is challenging to obtain an accurate settlement measurement. Therefore, we apply filtering technology to eliminate the influence of noise. Its mathematical expression is $y(k)=a x(k)-(1-a) y(k-1)$, where $a$ is the filter coefficient, $y(k)$ represents the current output, $x(k)$ represents the current input, and $y(k-1)$ represents the previous output. Figure 14 is the simulation result under the influence of vibration when the distance between the radar and track 1 is $1.0157 \mathrm{~m}$. The filtered track settlement is hardly affected by vibration, which allows us to try to apply this method to actual engineering.

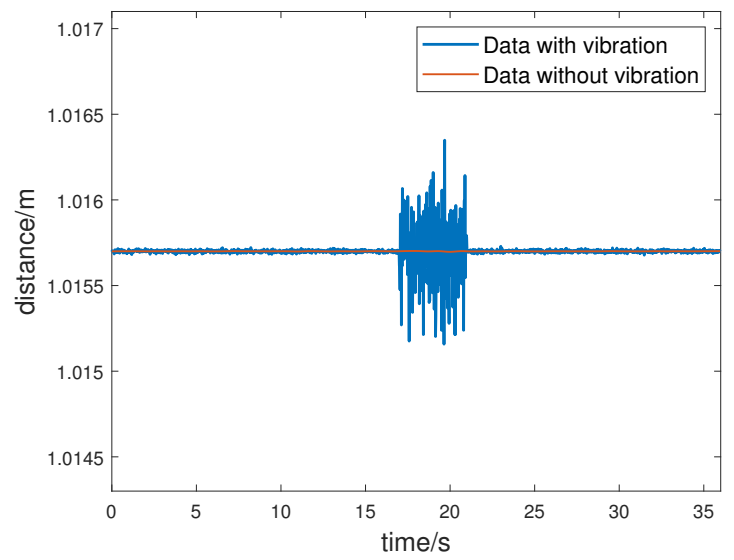

Figure 14. Experiment of track settlement measurement under simulated vibration signal. 


\section{Conclusions}

This paper presents a method for railway track settlement measurement based on the FMCW millimeter wave radar. Firstly, a data set of each object's RCS statistical features in the track settlement measurement environment is constructed. Then DAG-SVM classifier is trained to realize the track recognition based on this data set. The high-resolution frequency estimation algorithm of ACZT is used to realize the measurement of track settlement. The laboratory experiment platform we built verified the effectiveness of the proposed method. The experimental results show that the accuracy of track identification reached $95 \%$, and the accuracy of settlement measurement reached $0.5 \mathrm{~mm}$.

The method proposed in this paper is a successful attempt at the application of FMCW millimeter-wave radars in railway safety monitoring. On the premise of meeting the settlement measurement requirements, compared with manual measurement methods, the proposed method based on millimeter-wave radar has a higher degree of automation. Compared with machine vision methods, the proposed settlement measurement method is less affected by external conditions, which can work in low light and haze environments. However, as listed in Table 4, the proposed method has some engineering problems that need to be solved urgently. Therefore, in the next stage of work, we will combine the needs of settlement measurement and reasonably select multiple reference points and observation points for settlement measurement on the track line. Cooperating with related companies, we can optimize the installation and structural design of the radar so that the proposed method can meet the track settlement requirement in the actual railway environment.

Table 4. A list of the main engineering problems.

\begin{tabular}{ll}
\hline List & Problems and Possible Solutions \\
\hline \multirow{2}{*}{1} & P:Selection of base points and observation points \\
& S: Refer to manual measurement method \\
2 & P:Power supply \\
& S: Utilize solar energy and batteries \\
3 & P:Calibration of millimeter-wave radar \\
& S: Combination of automatic calibration and regular manual calibration \\
4 & P:Transfer of Settlement Detection Data \\
& S: Integration of Radar and Communication \\
\hline
\end{tabular}

Author Contributions: Conceptualization, S.L.; methodology, S.L. and W.L.; software, S.L. and J.D.; formal analysis, J.D.; resources, S.L.; data curation, S.L. and H.L.; writing-original draft preparation, S.L. and J.D.; writing-review and editing, S.L., J.D., H.L. and W.L.; visualization, F.Z. and Z.Z.; project administration, F.Z. and Z.Z.; funding acquisition, S.L. All authors have read and agreed to the published version of the manuscript.

Funding: This research is funded by National Natural Science Foundation of China(61502055); Hunan Science and Technology Plan Project (2016GK2003); the Research Foundation of Education Bureau of Hunan Province, China (Grant No.21B0303); Changsha Municipal Natural Science Foundation (Grant No. kq2014113) and the Hunan Provincial Department of Education under Award 18C0203.

Institutional Review Board Statement: Not applicable.

Informed Consent Statement: Not applicable.

Data Availability Statement: The data presented in this study are available on request from the corresponding author.

Conflicts of Interest: The authors declare no conflict of interest. 


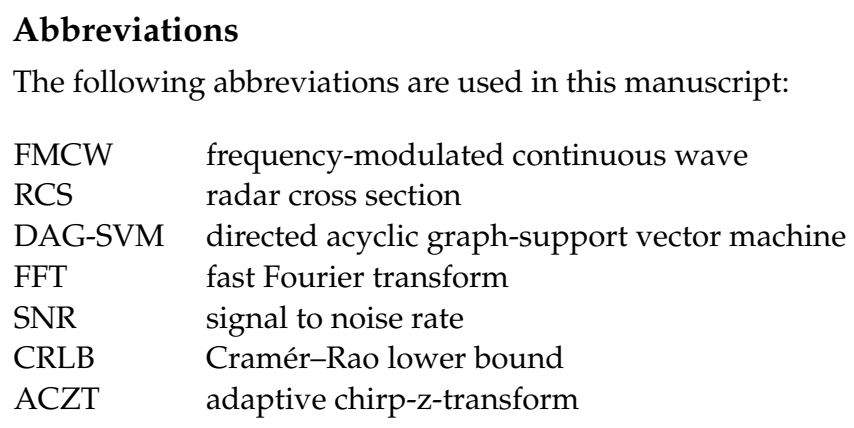

\section{References}

1. Chen, J.; Zhou, Y. Dynamic Responses of Subgrade under Double-Line High-Speed Railway. Soil Dyn. Earthq. Eng. 2018, 110, 1-12. [CrossRef]

2. Zou, J.; Zhu, Y.; Xu, Y.; Li, Q.; Meng, L.; Li, H. Mobile precise trigonometric levelling system based on land vehicle: An alternative method for precise levelling. Surv. Rev. 2017, 49, 249-258. [CrossRef]

3. Liu, B.; Lu, Z.; Chen, L.; Kong, W.; Sui, X. Accuracy Analysis of the InSAR Altimeter in Relative Elevation Measurements of the Sea Surface. IEEE Access 2021, 9, 27783-27789. [CrossRef]

4. $\quad$ Engels, G.; Aranjuelo, N.; Arganda, I.; Nieto, M.; Otaegui, O. 3D Object Detection from LiDAR Data using Distance Dependent Feature Extraction. arXiv 2020, arXiv:2003.00888.

5. $\quad$ Li, L.; Cao, X.Y.; Sun, Q.H.J.; Jia, B.S.; Dong, X. A new 3D laser-scanning and GPS combined measurement system. Comptes Rendus Geosci. 2019, 351, 508-516.

6. Chen, Y.F. Subgrade Settlement Monitoring System for High speed Railway Operation Line. Railw. Investig. Surv. 2017, 3, 28-31.

7. Li, Y.; Zhang, W.; Tian, B.; Lin, W.; Liu, Y. Scattering Model-Based Frequency-Hopping RCS Reconstruction Using SPICE Methods. Remote. Sens. 2021, 13, 3689. [CrossRef]

8. Wu, Q.; Chen, J.; Lu, Y.; Zhang, Y. A Complete Automatic Target Recognition System of Low Altitude, Small RCS and Slow Speed (LSS) Targets Based on Multi-Dimensional Feature Fusion. Sensors 2019, 19, 5048-5060. [CrossRef] [PubMed]

9. Patel, J.S.; Francesco, F.; David, A. Review of radar classification \& RCS characterisation techniques for small UAVs or drones IET Radar Sonar Navig. 2018, 12, 911-919.

10. Sasakawa, D.; Honma, N.; Nakayama, T.; Iizuka, S. Human Activity Identification by Height and Doppler RCS Information Detected by MIMO Radar. IEICE Trans. Commun. 2019, E102-B, 1270-1278. [CrossRef]

11. Lee, Y.; Choo, H.; Kim, S.; Kim, H. RCS based target recognition with real FMCW radar implementation. Microw. Opt. Technol. Lett. 2016, 58, 1745-1750. [CrossRef]

12. Wang, T.; Bi, W.J.; Zhao, Y.L.; Xue, W.C. Radar target recognition algorithm based on RCS observation sequence-Set-valued identification method. J. Syst. Sci. Complex. 2016, 29, 573-588. [CrossRef]

13. Lee, S.; Lee, B.; Lee, J.; Kim, S. Statistical Characteristic-Based Road Structure Recognition in Automotive FMCW Radar Systems IEEE Trans. Intell. Transp. Syst. 2019, 20, 2418-2429. [CrossRef]

14. Lee, J.; Kim, D.; Jeong, S.; Ahn, G.C.; Kim, Y. Target classification scheme using phase characteristics for automotive FMCW radar. Electron. Lett. Inst. Eng. Technol. 2016, 52, 2061-2063. [CrossRef]

15. Hyun, E.; Jin, Y.S.; Hwang, S.O. Human-vehicle classification scheme using doppler spectrum distribution based on 2D rangedoppler FMCW radar. J. Intell. Fuzzy Syst. 2018, 35, 6035-6045. [CrossRef]

16. Ding, C.; Chae, R.; Wang, J.; Zhang, L.; Hong, H.; Zhu, X.; Li, C. Inattentive Driving Behavior Detection Based on Portable FMCW Radar. IEEE Trans. Microw. Theory Tech. 2019, 67, 4031-4041. [CrossRef]

17. Ciattaglia, G.; Santis, A.; Disha, D.; Spinsante, S.; Castellini, P.; Gambi, E. Performance Evaluation of Vibrational Measurements through mmWave Automotive Radars. Remote Sens. 2021, 13, 98. [CrossRef]

18. Herzel, F.; Kissinger, D.; Ng, H.J. Analysis of Ranging Precision in an FMCW Radar Measurement Using a Phase-Locked Loop. Circuits Syst. I Regul. Pap. IEEE Trans. 2017, 65, 783-792. [CrossRef]

19. Yamaguchi, K.; Saito, M.; Akiyama, T.; Kobayashi, T.; Matsue, H. A 24 GHz band FMCW radar system for detecting closed multiple targets with small displacement. In Proceedings of the 2015 Seventh International Conference on Ubiquitous and Future Networks, Sapporo, Japan, 7-10 July 2015.

20. Piotrowsky, L.; Jaeschke, T.; Kueppers, S.; Siska, J.; Pohl, N. Enabling High Accuracy Distance Measurements With FMCW Radar Sensors. IEEE Trans. Microw. Theory Tech. 2019, 67, 5360-5371. [CrossRef]

21. Scherr, S.; Ayhan, S.; Fischbach, B.; Bhutani, A.; Pauli, M.; Zwick, T. An Efficient Frequency and Phase Estimation Algorithm With CRB Performance for FMCW Radar Applications. IEEE Trans. Instrum. Meas. 2015, 64, 1868-1875. [CrossRef]

22. Scherr, S.; Afroz, R.; Ayhan, S.; Thomas, S.; Jaeschke, T.; Marahrens, S.; Bhutani, A.; Pauli, M.; Pohl, N.; Zwick, T. Influence of Radar Targets on the Accuracy of FMCW Radar Distance Measurements. IEEE Trans. Microw. Theory Tech 2017, 65, 3640-3647. [CrossRef]

23. Arab, H.; Dufour, S.; Moldovan, E.; Akyel, C.; Tatu, S.O. A 77-GHz Six-Port Sensor for Accurate Near-Field Displacement and Doppler Measurements. Sensors 2016, 18, 2565-2584. [CrossRef] 
24. Bhutani, A.; Marahrens, S.; Gehringer, M.; Göttel, B.; Pauli, M.; Zwick, T. The Role of Millimeter-Waves in the Distance Measurement Accuracy of an FMCW Radar. Sensors 2019, 19, 3938-3954. [CrossRef]

25. Ioffe, A.; Doerr, W.; Yan, H.H.; Vu, D.H.; Arage, A.H. RCS characteristics of street curbs and the applications in automotive radar classification. In Proceedings of the 2016 European Radar Conference (EuRAD), London, UK, 5-7 October 2016.

26. Ünald1, S.; Bodur, H.; Çimen, S.; Çakır, G. RCS reduction of reflectarray using new variable size FSS method. AEU Int. J. Electron. Commun. 2020, 117, 153098. [CrossRef]

27. Jing, S. Target identity recognition method based on RCS distribution and spatial location. Procedia Comput. Sci. 2019, 147, 632-637. [CrossRef]

28. Liu, G.; Yang, C.; Liu, S. Xiao, C.; Song, B. Feature Selection Method Based on Mutual Information and Support Vector Machine. Int. J. Pattern Recognit. Artif. Intell. 2021, 35, 2150021. [CrossRef]

29. Chang, C.Y.; Chang, C.W.; Kathiravan, S.; Lin, C.; Chen, S.T. DAG-SVM based infant cry classification system using sequential forward floating feature selection. Multidim Syst. Sign Process 2017, 28, 961-976. [CrossRef]

30. Zhang, C.; Qing, A.; Meng, Y. The Application of Chirp Z-Transform in Fast Computation of Antenna Array Pattern. Appl. Comput. Electromagn. Soc. J. 2019, 34, 1685-1693.

31. Sampath, A.K.; Gomathi, D.N. Decision tree and deep learning based probabilistic model for character recognition. J. Cent. South Univ. 2017, 24, 2862-2876. [CrossRef]

32. Hu, C.; Wu, Y.; Huang, L.; Yan, G. Unitary root-MUSIC based on tensor mode-R algorithm for multidimensional sinusoidal frequency estimation without pairing parameters. Multidim Syst. Sign Process 2020, 31, 491-501. [CrossRef]

33. Lee, J.S.; Yoon, T.M.; Lee, K.B. Bearing fault detection of IPMSMs using zoom FFT. J. Electr. Eng. Technol. 2016, 11, 1235-1241. [CrossRef]

34. Wang, H.K.; Cao, D.X.; Liu, Y.Q.; Wang, T. Vibration test analysis of the curved track model under train operation. Chin. J. Appl. Mech. 2020, 37, 701-706. 